\title{
Macromycetes in the Dendrological Park of the Warsaw Agricultural University
}

\author{
ANDRZEJ SZCZEPKOWSKI \\ Department of Mycology and Forest Phytopathology, Warsaw Agricultural University \\ Nowoursynowska 159, PL-02-776 Warszawa, szczepkowski@delta.sggw.waw.pl
}

Szczepkowski A.: Macromycetes in the Dendrological Park of the Warsaw Agricultural University. Acta Mycol. 42 (2): 179-186, 2007.

A list of 79 species of macromycetes recorded in the Dendrological Park of the Warsaw Agricultural University in Warsaw between 1996 and 2005 is discussed in the paper.

Key words: macromycetes, urban area, park, conservation of fungi, Poland

\section{INTRODUCTION}

Warsaw is one of the few Polish urban centres where mycological examinations have been conducted, and its ecology has been investigated. Similarly to other agglomerations in Poland, however, the knowledge on marcomycetes occurring in it is unsatisfactory (Adamczyk, Ławrynowicz 1991; Zimny 2000). Studies by Chełchowski $(1888,1898,1902)$ and Błoński $(1890,1896)$ with records of 467 fungal species in the city at the time, are of fundamental importance for contemporary comparative studies on the occurrence of fungi in Warsaw. Skirgiełło and Domański (1981), and Ławrynowicz (1982), who noticed a decrease in the number of species of parasitic and mycorrhizal fungi in the city centre, greatly contributed to the study of macromycetes in towns.

In urban areas, parks play an important role of habitats for many species, including fungi for which they are often the only natural sites (Lawrynowicz 1990). Although there are over 80 parks and gardens in Warsaw, systematic observations of macromycetes were published only from the Botanical Garden of the University of Warsaw. A total number of 58 species was collected in the entire area of the Garden (ca. 4.5 ha) over three years of studies (Szober 1965).

The aim of this study is to present the species composition of macromycetes noted in the Dendrological Park of the Warsaw Agricultural University and to define the Park's role as the environment of macromycetes in Warsaw. 


\section{STUDY AREA AND METHODS}

The Dendrological Park is a fenced site located along the Rakowiecka street in the district of Mokotów in the city centre (Fig. 1). Established as a botanical and dendrological garden in the 1920s, it initially covered 0.81 ha (Kęsick a 1932). The Mokotów fields, where it was set up, served as the ground for military exercises and horse races over a few decades (Rewerska 1937). The garden covered over 2.25 ha in 1937, and comprised three sections: systematic, dendrological, and pomological (St aff 1937). Its part was later reclaimed for the construction of one of the adjoining streets (al. Niepodległości) and buildings of the Warsaw School of Economics (Za rę ba 1977). The Park was neglected after the II World War, and it stayed so until the 1970s when its supervision was reassigned to what now is the Department of Forest Botany, Warsaw Agricultural University. The Park was revitalised and its area was redeveloped. Five hundred and seventeen specimens of trees and shrubs were recorded in the Park survey conducted at the time (Rodek 1976). The Park was enriched with new tree and shrub species or varieties, both native and introduced. The planting material was derived from the Arboretum of the Warsaw Agricultural University in Rogów. Filipczak (1998) reports that 522 trees and shrubs belonging to 99 species and varieties grow in the park. Some of them, such as Fagus sylvatica var. atropurpurea or Conylus colurna, were considered to be nature monuments. In 2003, the Park, covering 1.65 ha, became legally protected as a nature and landscape complex. Taxa belonging to angiosperms (Angiospermae) prevail among trees and shrubs (Fig. 2). Specimens of such genera as Acer, Aesculus, Betula, Fraxinus, Populus, Quercus, Robinia, Tilia occur most numerously. Shrubs include Berberis, Laburnum, Ribes, Spiraea, Symphoricarpos, Syringa. Gymnosperms (Gymnospermae) represent such genera as Ginkgo, Chamaecyparis, Larix, Pinus, Taxus, Thuja. The layer of

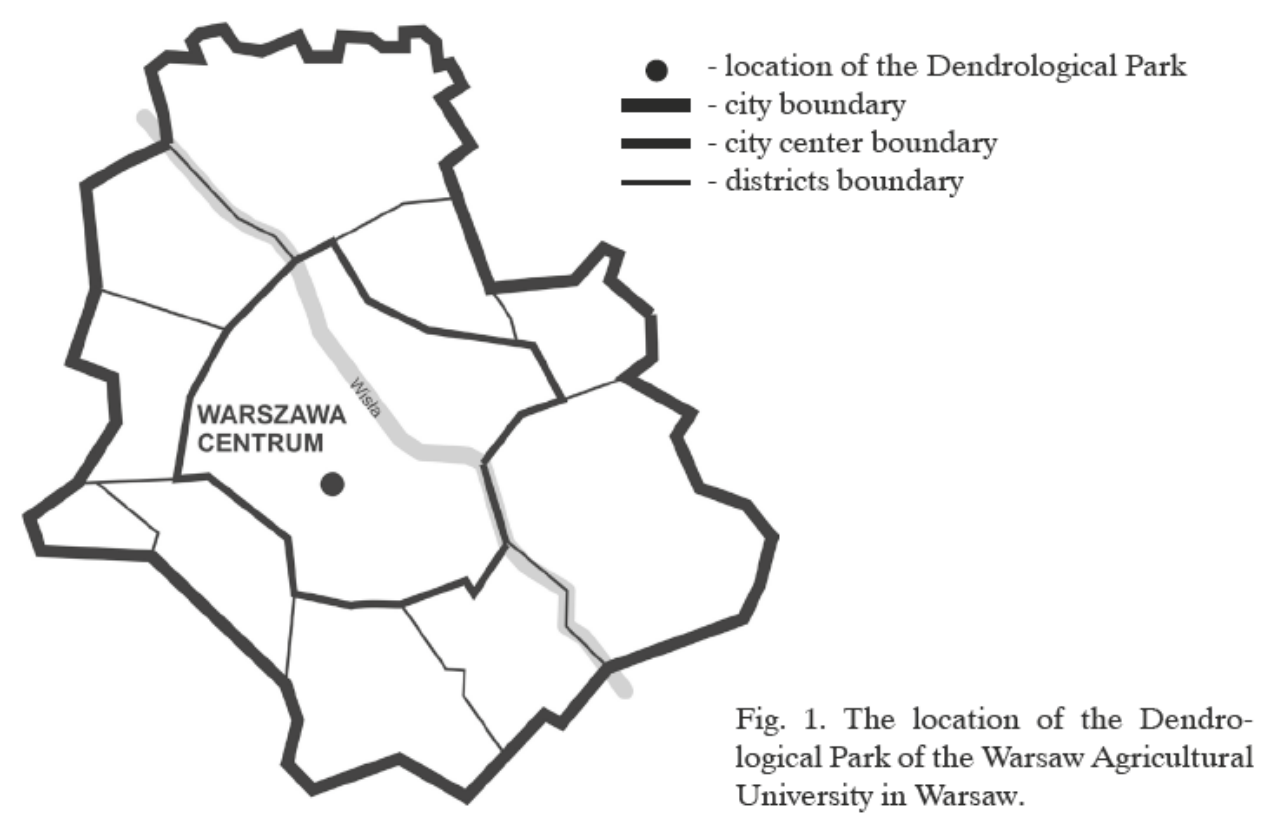


herbaceous vegetation consists of such plants as Aegopodium podagraria, Anemone nemorosa, Ficaria verna, Gagea lutea, Galeobdolon luteum, Galium aparine, Impatiens parviflora, Lamium album, Urtica dioica, Plantago lanceolata, Symphytum officinale, Taraxacum officinale, Trifolium repens, Viola spp. According to Zi e lo ny (2005), elements of the typical oak-lime-hornbeam community, Tilio-Carpinetum typicum, may be found in the Park. A poorly preserved network of alleys and paths is still identifiable. A small cemented water reservoir is situated in the central part of the Park. The field layer and lawns are mown every year, and leaves and fallen branches are raked in the autumn. Tree-stand maintenance work was conducted in 1993 and 1997. Tree crowns were thinned, dead limbs and branches were cut, and injures were treated. Dead or dying trees and shrubs, self-sown seedlings as well as trees or shrubs constituting a risk of damage to the fence and the adjacent University buildings, were felled. Because of progressing tree dying (Fig. 3), Tu miłowicz (1993) emphasised the need to complement the existing tree-stand with new plantings as early as in 1993. Over a decade later, his demands have not been acted upon.

Although the Park has been an important teaching site and an experimental field for a number of faculties of the Warsaw Agricultural University since it was established (Staff 1937; Zaręba 1977), no research study discussing it has been published so far.

The present list of macromycetes is the result of observations conducted between 1996 and 2005. Systematic observations were conducted at least once every month in the vegetative seasons between 1998 and 2003. The Park was visited sporadically outside this period. A thorough survey of the entire area could not be conducted on each visit.

The recorded fungi were classified in three basic ecological groups: terrestrial species - i.e. fungi occurring on soil, both saprotrophic and mycorrhizal; litter-decomposing species - i.e. saprotrophs growing on litter (fallen leaves, fruits and little twigs); lignicolous species - i.e. fungi occurring saprotrophically on wood or parasitizing live trees.

The collected fungi were determined using mainly the following monographs and keys: Hansen and Knudsen (1992, 1997, 2000), Ryvarden and Gilbertson (1993, 1994), Rudnicka - Jezierska (1991), Skirgiełło $(1991,1998)$ and Gumińska (1997). The systematic division of fungi follows Kirk at al. (2001). Species names of the Ascomycetes are given according to Hansen and Knudsen (2000) while the nomenclature of the Basidiomycetes follows Wojew oda (2003).

\section{RESULTS}

\section{LIST OF SPECIES}

Explanations: 1 - litter decomposing species; $1 \mathrm{lg}$ - lignicolous species; $\mathrm{t}$ - terrestrial species; $\mathbf{P}$ - protected species; $\mathbf{I}$ - indeterminate (category) species; $\mathbf{R}$ - rare species, $\mathbf{V}$ - vulnerable species

Agaricus bitorquis (Quél.) Sacc. - t, 05 1998, 06 2000, 052001.

A. arvensis Schaeff. - t, 07 1998-2000, 09-10 2000, 08 2001, 09 2002, 102003.

Armillaria mellea (Vahl: Fr.) P. Kumm. ss. lato. - $\lg$ (on roots of Robinia), 092003. 
Auricularia auricula-judae (Bull.: Fr.) Wettst. - $\lg$ (on branches and trunks of Acer negundo, Fraxinus excelsior, Ptelea trifoliata, Sambucus nigra), 09 1997, 03 1998, 10-11 1998, 0912 2000, 10 2003, 032005.

Auriculariopsis ampla (Lév.) Maire - $\lg$ (on dead branch of Populus), 102005.

Bjerkandera adusta (Willd.: Fr.) P. Karst. - $\lg$ (on stumps and trunks of Acer, Fagus, Fraxinus, Quercus), 04-11 1998-2003, 09 2004, 072005.

Boletus luridus Schaeff.: Fr. - t (in neighborhood of Acer, Aesculus, Quercus), 07 2004.

Calocybe gambosa (Fr.) Donk - t, 05 2001, 2004.

Chondrosterum purpureum (Schum.: Fr.) Pouzar - $\lg$ (on trunks and stumps of Acer and Populus), 09-10 2001-2003, 102004.

Coniophora puteana (Schum.: Fr.) P. Karst. - $\lg$ (on rotten log of Tilia), 10 2002, 11 2003.

Coprinus atramentarius (Bull.: Fr.) Fr. - t, 06 1999, 07, 11 2000, 10 2002, 09 2003, 05, 062004.

C. comatus (Müll.: Fr.) Pers. - t, 09 2001, 102005.

C. disseminatus (Pers.: Fr.) - $\lg$ (on and around stumps and living Populus), 062001 , 052003.

C. micaceus (Bull.: Fr.) Fr. - $\lg$ (on and around stumps); 06 1999, 05, 09 2001, 04, 05 2004.

C. plicatilis (Curt.: Fr.) Fr. - t (among grass), 07 2002, 052003.

Entoloma euchroum (Pers.: Fr.) Donk - $\lg$ (on stump and trunk of Acer tataricum and Fraxinus ornus), 09 2003, 2004, 10 2004; I.

Exidia plana (Wiggers) Donk - lg (on lying branches of deciduous trees), 11 2001, $092002,032003$.

Flammulina velutipes (M. A. Curtis: Fr.) Singer $-\lg$ (on branches and trunks of Acer, Juglans, Tilia), 09 1997, 11 2000, 03, 112003.

Fomes fomentarius (L.: Fr.) Kickx - lg (on dead trunk of Populus), 061997.

Ganoderma adspersum (S. Schulz.) Donk - $\lg$ (on stump of deciduous tree), 04 2002, 082005.

G. applanatum (Pers.) Pat. - $\lg$ (on stumps and trunks of Acer, Castanea, also on lying log of Tilia ), 08 1998, 05-09 2000-2003, 102004.

G. pfeifferi Bres. - $\lg$ (on stump of Acer saccharinum), 2001-2002 (S zcze p k ow sk i, Piętk a 2003).

Geastrum coronatum Pers. sensu P hillips (1981) - t (under Syringa josikaea); 0809 2003, 08 2004; P, V; (det. W. Wojewoda).

Gymnopus dryophilus (Bull.: Fr.) Murrill - t, 07 2000, 06, 092003.

Helvella acetabulum (L.: Fr.) Quél. - t (among grass and mosses), 05 1999, 06-07 2004.

Hygrocybe conica (Schaeff.: Fr.) P. Kumm. - t (among grass), 06 2003; 072004.

Hymenoscyphus fructigenus (Bull.: Fr.) Gray - 1 (on acorns), 09 2002, 102004.

Hypoxylon fragiforme (Pers.: Fr.) J. Kickx - $\lg$ (on dead branch of Fagus sylvatica), 091996.

Inocybe fastigiata (Schaeff.) Quél. - t; 07 1999-2000, 06 2002-2003.

Inonotus radiatus (Sowerby: Fr.) P. Karst. - lg (on stump of Alnus), 102003.

Laccaria laccata (Scop.: Fr.) Berk. \& Broome - t (among grass), 07 1999, 092003.

Lactarius quietus (Fr.) Fr. - t (in neighbourhood of Quercus), 07 2000, 092003. 
Langermannia gigantea (Batsch.: Pers.) Rostk. - t; 07 1996, 07 1997, 06-09 1998, 07 1999-2000, 08 2003; P (Szcze pkowski 2002).

Lepiota aspera (Pers.: Fr.) Quél. - t (among grass), 092003.

L. cristata (Bolt.: Fr.) P. Kumm. - t (among grass), 07 2000-2001, 092003.

Lepista nuda (Bull.: Fr.) Cooke - t (near compost heap), 10-11 2000.

Lyophyllum decastes (Fr.: Fr.) Singer - t, 092003.

Macrolepiota rhacodes (Vittad.) Singer - t (on compost heap and also among grass), 07, 09 2000, 07 2002, 07, 09 2003, 08 2004; I.

Marasmius rotula (Scop.: Fr.) Fr. - l (on lying twigs), 07 1998, 06 1999, 062004.

Meripilus giganteus (Pers.: Fr.) P. Karst. - lg (on stump of Fagus sylvatica), 07-10 19992005; P (Szczepkowski 2002).

Morchella semilibera DC.: Fr. - t (under Populus), 04 2003, 05 2004; P, I.

Mycena galericulata (Scop.: Fr.) S. F. Gray - $\lg$ (on stumps of deciduous trees), 06 1999, 07 2000, 09 2003, 102004.

Nectria cinabarina (Tode) Fr. - lg (on lying branches of deciduous trees), 032001 , 052003.

Paxillus involutus (Batsch: Fr.) Fr. - t, 06-08 1999, 07, 09 2000, 08 2001, 06, 10 2003, 072004.

Peniophora quercina (Pers.: Fr.) Cooke - lg (on dead branches of Quercus), 121998 , 09 2003, 09 2004, 102005.

Perenniporia fraxinea (Bull.: Fr.) Ryv. - lg (at the base of Populus), 08 2003, 102004.

Phallus hadrianii Vent.: Pers. - t, 06-08 1996-2003, 06 2004; P (S zczepkowski 2002).

Phellinus igniarius (L.: Fr.) Quél. - lg (on branch of Salix fragilis), 092004.

Ph. robustus (P. Karst.) Bourdot \& Galzin - Ig (on trunk of Quercus robur), Jan-12 1998-2005.

Ph. pomaceus (Pers.) Maire - lg (on branch of Prunus), 01-12 2000-2003.

Phlebia radiata Fr. - lg (on dead branch of Sorbus), 09 2002, 112003.

Ph. tremellosa (Schrad.: Fr.) Nakasone \& Burds. - lg (on lying log of Tilia), 09-11 2002-2003.

Pholiota aurivella (Batsch.: Fr.) P. Kumm. - Ig (on trunk of Acer platanoides), 11 2003.

Ph. populnea (Pers.: Fr.) Kuyper \& Tjall. - lg (on trunk of Populus), 092000.

Ph. squarrosa (Weigel: Fr.) P. Kumm. - lg (at the base of trunk of Acer tataricum), 10 2003-2004.

Piptoporus betulinus (Bull.: Fr.) P. Karst. - lg (on dead branches of Betula), 01-12 2001-2003, 09 2004, 09-10 2005.

Pleurotus dryinus (Pers.: Fr.) P. Kumm. - $\lg$ (on trunk of living Sorbus), 09 2003; R.

P. ostreatus (Jacq.: Fr.) P. Kumm. - lg (on trunk of Juglans), 102002.

Pluteus atricapillus (Batsch) Faylod - lg (on stumps of deciduous trees), 07 1998, 05 2001, 09 2003, 06 2004, 102005.

Polyporus squamosus (Huds.) Fr. - lg (on stumps and trunks of Acer, Salix, Juglans), 04-09 1997-2004.

Psilocybe fascicularis (Huds.: Fr.) Noordel. - $\lg$ (on stumps of deciduous trees), 10 1997, 09 1998, 07-09 2000, 07 2001, 09 2002, 072004.

Ramaria stricta (Pers.: Fr.) Quél. - t (on rotten stump of Populus), 092002.

Russula foetens (Pers.: Fr.) Fr. - t, 072000. 
Sarcodontia crocea (Schwein.: Fr.) Kotl. - $\lg$ (on branch of Malus), 09 2003; R.

Schizophyllum commune Fr.: Fr. - $\lg$ (on branches and trunks of Acer, Fagus, Tilia), 04-11 1998-2003.

Scleroderma verrucosum (Bull.): Pers. - t (in neighborhood of Acer, Aesculus, Quercus), 07-08 2001, 09 2002, 07, 09 2003, 072004.

Scutellinia scutellata (L.: Fr.) Lambotte - $\mathrm{t}$ (on rotten stump of Fagus sylvatica), 05-07 2001, 04 2002, 06-07 2003, 052004.

Stereum hirsutum (Willd.: Fr.) Gray - $\lg$ (on stumps, trunks and branches of Acer, Aesculus, Quercus), 08-09 1996, 09 1998, 06-11 2000, 09-10 2003.

Suillus grevillei (Klotzsch: Fr.) Singer - t (under Larix decidua), 07-08 2000.

Trametes hirsuta (Wulf.: Fr.) Pilát - $\lg$ (on lying branch of Populus and Betula), 09-11 2003, 10 2004-2005.

T. gibbosa (Pers.: Fr.) Fr. - lg (on trunk of Acer platanoides), 102005.

Tyromyces fissilis (Berk. \& M. A. Curtis) Donk - $\lg$ (on trunk of Acer tataricum), 0809 2002; R.

Ustulina deusta (Hoffm.: Fr.) Lind - $\lg$ (on stump of deciduous tree), 06 2002, 08 2004.

Volvariella bombycina (Schaeff.: Fr.) Singer - $\lg$ (on trunks of living Acer negundo, Acer platanoides, Fraxinus excelsior), 06-07 2002-2003, 08 2004; I.

V. pusilla (Pers.: Fr.) Quél. - t (among grass), 07 2004; I.

Xerocomus rubellus (Krombh.) Quél. - t (in neighborhood of Quercus, Tilia, Acer), 07 2000, 06 2001, 072002.

X. pascuus (Pers.) Krombh. - t, 06-08 2000, 06-07 2003.

Xylaria hypoxylon (L.) Grev. - $\lg$ (on stumps of deciduous trees), 09 2000, 11 2003, 052004.

X. polymorpha (Pers.) Grev. - $\lg$ (on buried rotten bits of deciduous wood and on stump of Fagus sylvatica), 07 2000, 2001, 08-09 2003, 102004.

\section{FINAL REMARKS}

1. A total number of 79 species of macromycetes was recorded in the Dendrological Park of the Warsaw Agricultural University, mostly the Basidiomycetes $(88.6 \%)$.

2. Lignicolous species were the dominating ecological group (58.3\% of all species). Terrestrial species and litter-decomposing species constituted $39.2 \%$ and $2.5 \%$, respectively.

3. Nine species, that is $11.4 \%$ of the fungi examined, are red-listed in Poland (Wojewoda, Ławrynowicz 2006): Entoloma euchroum, Geastrum coronatum, Morchella semilibera, Macrolepiota rhacodes, Pleurotus dryinus, Sarcodontia crocea, Tyromyces fissilis, Volvariella bombycina, Volvariella pusilla and five species of strictly protected fungi in Poland: Geastrum coronatum (Fig. 4), Langermannia gigantea (Fig. 5), Meripilus giganteus (Fig. 6), Morchella semilibera (Fig. 7), Phallus hadrianii (Fig. 8) were recorded in the Park.

4. The locality of Perenniporia fraxinea (Fig. 9) in the Park is its second published occurrence site in Warsaw and, at the same time, in Poland (Szcze pkowski 2004), while Populus is a new host in the Polish population of this rare fungus. 
5. The results show that the Dendrological Park of the Warsaw Agricultural University in the city centre is an important natural site of many macromycetes, including a few species protected in Poland.

Acknowledgement. My special thanks go to Prof. Anna Bujakiewicz (Poznań) for her valuable remarks and comments on the first draft of the manuscript, Prof. Władysław Wojewoda for the determination of Geastrum coronatum, Prof. Stefan Zajączkowski (Warsaw) for providing access to the Park documentation deposited at the Department of Forest Botany, Warsaw Agricultural University, Dr. Anna Różańska (Warsaw) for her kind help in finding information and literature on the Park.

\section{REFERENCES}

Adamczyk J., Ławrynowicz M. 1991. Stan zbadania grzybów wielkoowocnikowych miast Polski. Wiad. Bot. 35 (1): 3-9.

Błońs ki F. 1890. Wyniki poszukiwań florystycznych skrytokwiatowych, dokonanych w ciągu lata r. 1889 w obrębie 5-ciu powiatów Królestwa Polskiego. Pam. Fizjogr. 10 (3): 129-190.

Błońsk i F. 1896. Przyczynek do flory grzybów Polski. Pam. Fizjogr. 14 (3): 63-93.

Ch ełch ow sk i S. 1888. Basidialnyje griby okrestnostnej Varšavy. Varš. Univ. Izv. Warszawa. 112 pp.

Chełchowski S. 1898. Grzyby podstawkozarodnikowe Królestwa Polskiego. I. Autobasidiomycetes. Pam. Fizjogr. 15 (3): 3-285.

Ch ełch ows ki S. 1902. Spostrzeżenia grzyboznawcze. Pam. Fizjogr. 17 (3): 3-38.

Gumińska B. 1997. Flora Polska. Grzyby (Mycota) 26: Wodnichowate (Hygrophoraceae). PWN, Warszawa-Kraków.

Hansen L., Knudsen H. (eds). 1992. Nordic macromycetes. 2. Polyporales, Boletales, Agaricales, Russulales. Nordsvamp-Copenhagen.

Hansen L., Knudsen H. (eds). 1997. Nordic macromycetes. 3. Heterobasidioid, Aphyllophoroid and Gastromycetoid Basidiomycetes. Nordsvamp-Copenhagen.

Hansen L., Knudsen H. (eds). 2000. Nordic macromycetes. 1. Ascomycetes. Nordsvamp-Copenhagen.

Filipczak R. 1998. Multimedialny system informacji przestrzennej dla ogrodu dendrologicznego SGGW przy ulicy Rakowieckiej. Praca magisterska, Wydział Leśny SGGW, Warszawa.

Kęsicka I. 1932. Projekt otoczenia zieleńcowego i ogrodu botanicznego wokół gmachów SGGW przy ul. Rakowieckiej. Praca dyplomowa z Architektury Krajobrazu i Parkoznawstwa, Skierniewice.

Kirk P. M., Cann on P. F., David J. C., Stalpers J. C. 2001. Ainsworth and Bisby's. Dictionary of the Fungi. 9 ed. CAB International, Biddles Ltd, UK.

Ław ry nowicz M. 1982. Macrofungal flora of Łódź (In:) R. Bornka m m, J. A. Lee, M. R. D. Se a w a r d (eds). Urban Ecology. The Second European Ecological Symposium. Berlin 8-12 September. Blackwell Scientific Publications. Oxford, London. Edinburgh, Boston, Melbourne: 41-47.

Ławrynowicz M. 1990. Miasto jako środowisko życia dla grzybów makroskopowych na przykładzie Łodzi. (In:) Problemy ochrony i kształtowania środowiska przyrodniczego na obszarach zurbanizowanych. Centralny Program Badań Podstawowych 04. 10. Ochrona i Kształtowanie Środowiska Przyrodniczego. Vol. 22. Wyd. SGGW-AR, Warszawa.

Phillips R. 1981. Mushrooms and other Fungi of Great Britain and Europe. Pan Books Ltd, London.

Rew e r s k a L. 1937. Opis i uzasadnienie projektu dyplomowego pt. „Otoczenie parkowe gmachów Szkoły Głównej Handlowej w Warszawie". Praca magisterska, Wydział Ogrodniczy SGGW, Warszawa.

Rudnicka-Jezierska W. 1991. Flora Polska. Grzyby (Mycota) 23: Purchawkowate (Lycoperdales), Tęgoskórowe (Sclerodermatales), Pałeczkowate (Tulostomatales), Gniazdnicowate (Nidulariales), Sromotnikowe (Phallales), Osiakowe (Podoxales). PWN, Warszawa-Kraków.

Rodek L. A. 1976. Zastosowanie tachimetru Daltha 020 do inwentaryzacji parków. Praca magisterska, Wydział Leśny SGGW, Warszawa.

Ryvarden L., Gilberts on R. L. 1993. European polypores. 1. Abortiporus-Lindtneria. Synopsis Fungorum 6. Fungiflora, Oslo.

Ryvarden L., Gilberts on R. L. 1994. European polypores. 2. Meripilus-Tyromyces. Synopsis Fungorum 7. Fungiflora, Oslo. 
St aff F. (ed.) 1937. Księga Pamiątkowa Ku uczczeniu potrójnej rocznicy zaczątków, założenia, utrwalenia Szkoły Głównej Gospodarstwa Wiejskiego w Warszawie (1906-1911-1916-1936). Wydana staraniem Senatu Akademickiego Szkoły.

Skirgiełło A. 1965-1984. Materiały do rozmieszczenia geograficznego grzybów wyższych w Europie. I-VI. Acta Mycol.1: 23-26, 3: 243-249, 6: 101-123, 8 (2): 191-218, 12 (2): 155-189, 20 (1): 129-157.

Skirgiełło A. 1991. Flora Polska. Grzyby (Mycota) 20: Gołąbek (Russula). PWN, Warszawa-Kraków.

Skirgiełło A. 1998. Flora Polska. Grzyby (Mycota) 25: Mleczaj (Lactarius). PWN, Warszawa-Kraków.

Sk ir gi ełło A., D o m ań s k i Z.1981. Higher fungi of the central part of Warsaw. (In:) M. S e m e r dži e va and V. Š a š ek (eds). Organizace boje proti otrávam houbami v ČSSR a Polsku: 40-44. Komise pro mykologickou toxikologii Čsl. vědecké společnosti pro mykologii při ČSAV, Praha.

Szczepkowski A. 2002. Grzyby chronione w parku SGGW. Agricola 52: 30-31.

Szczepkowski A., Pięt ka J. 2003. New localities and new host of Ganoderma pfeifferi Bres. in Pat. in Poland. Acta Mycol. 38 (1/2): 55-59.

Szcze pkowski A. 2004. Perenniporia fraxinea (Fungi, Polyporales), a new species for Poland. Polish Botanical Journal 49 (1): 73-77.

Sz o b e r J. 1965. Grzyby wyższe Ogrodu Botanicznego Uniwersytetu Warszawskiego. Z obserwacji prowadzonych w latach 1961-1963. PWN, Warszawa.

Tu miło w i c z J. 1993. Sprawozdanie z wykonanych zabiegów pielęgnacyjno-chirurgicznych przy drzewostanie w Arboretum SGGW przy ul. Rakowieckiej w Warszawie. Maszynopis, Katedra Botaniki Leśnej SGGW, Warszawa, 2 pp.

Woje wo d a W. 2003. Checklist of Polish Larger Basidiomycetes. W. Szafer Institute of Botany, Polish Academy of Sciences, Kraków.

Wojewoda W., Lawrynowicz M. 2006. Red list of macrofungi in Poland. (In:) Z. Mirek, K. Zarzycki, W. Wojewoda, Z. Szeląg (eds). Red list of plants and fungi in Poland. W. Szafer Institute of Botany, Polish Academy of Sciences, Kraków: 50-70.

Zaręb a R. 1977. Zabytkowy park. Życie Warszawy (Życie i Nowoczesność) nr 346, 06.011977.

Zielony R. 2005. Park SGGW. (In:) J. Woj t a tow icz (ed.). Warszawska przyroda. Obszary i obiekty chronione. Biuro Ochrony Środowiska Urzędu m. st. Warszawy, Warszawa.

Zim ny H. 2000. Realizacja ochrony środowiska w Polsce. (In:) Ochrona przyrody i środowiska w Polsce oraz udział w niej organizacji społecznych. LOP, Warszawa.

\section{Macromycetes Parku Dendrologicznego SGGW w Warszawie}

\section{Streszczenie}

Przedstawiono skład gatunkowy grzybów wielkoowocnikowych Parku Dendrologicznego SGGW położonego przy ul. Rakowieckiej na Mokotowie w centrum Warszawy. Obserwacje prowadzono w latach 1996 - 2005. Stwierdzono występowanie 79 gatunków macromycetes. Dominowały Basidiomycetes $-88,6 \%$, a Ascomycetes stanowiły 11,4\%. Pięć gatunków podlega ścisłej ochronie prawnej, a 9 gatunków umieszczone jest na czerwonej liście grzybów zagrożonych w Polsce. Park Dendrologiczny SGGW stanowi ważną ostoję w centrum Warszawy dla wielu gatunków grzybów wielkoowocnikowych, w tym dla kilku podlegających prawnej ochronie gatunkowej. 


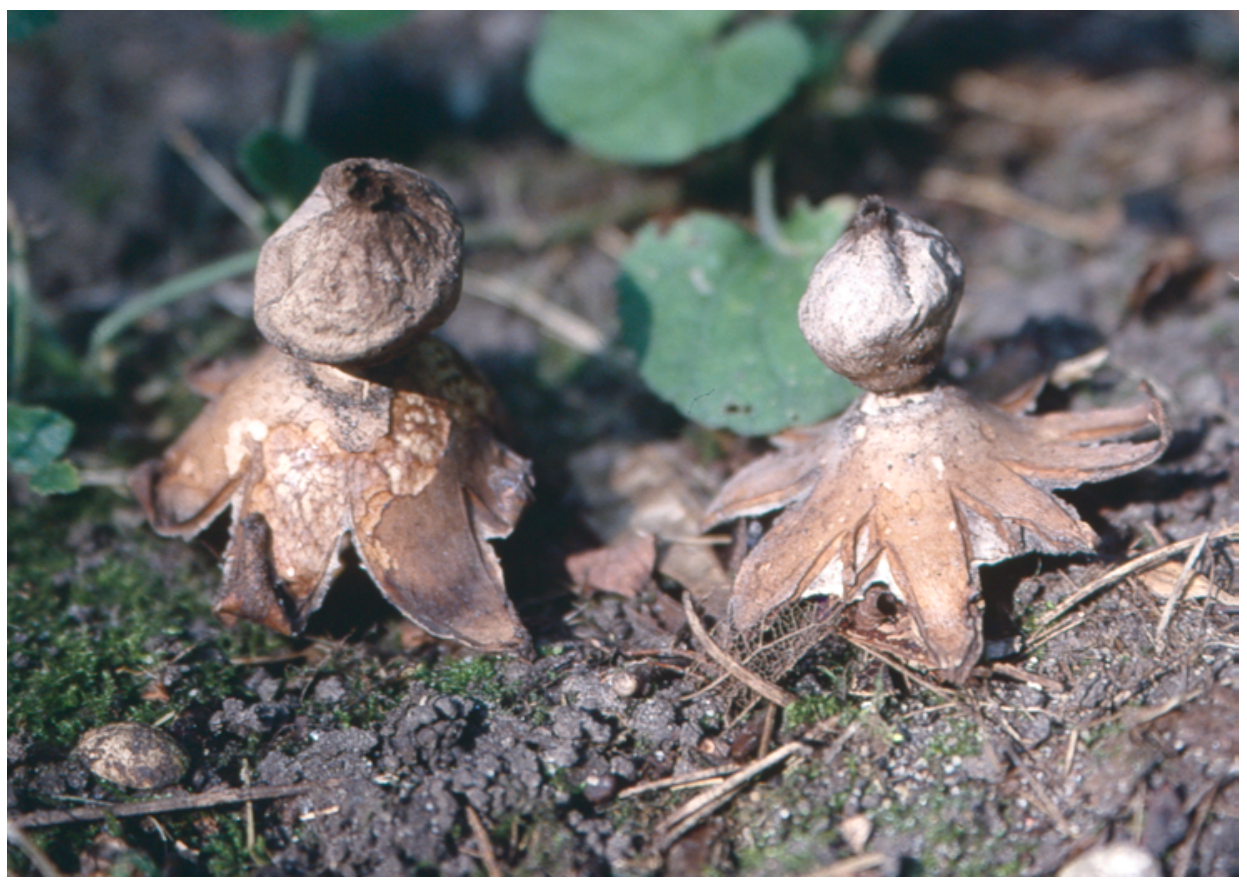

Fig. 4. Geastrum coronatum. Phot. A. Szczepkowski.

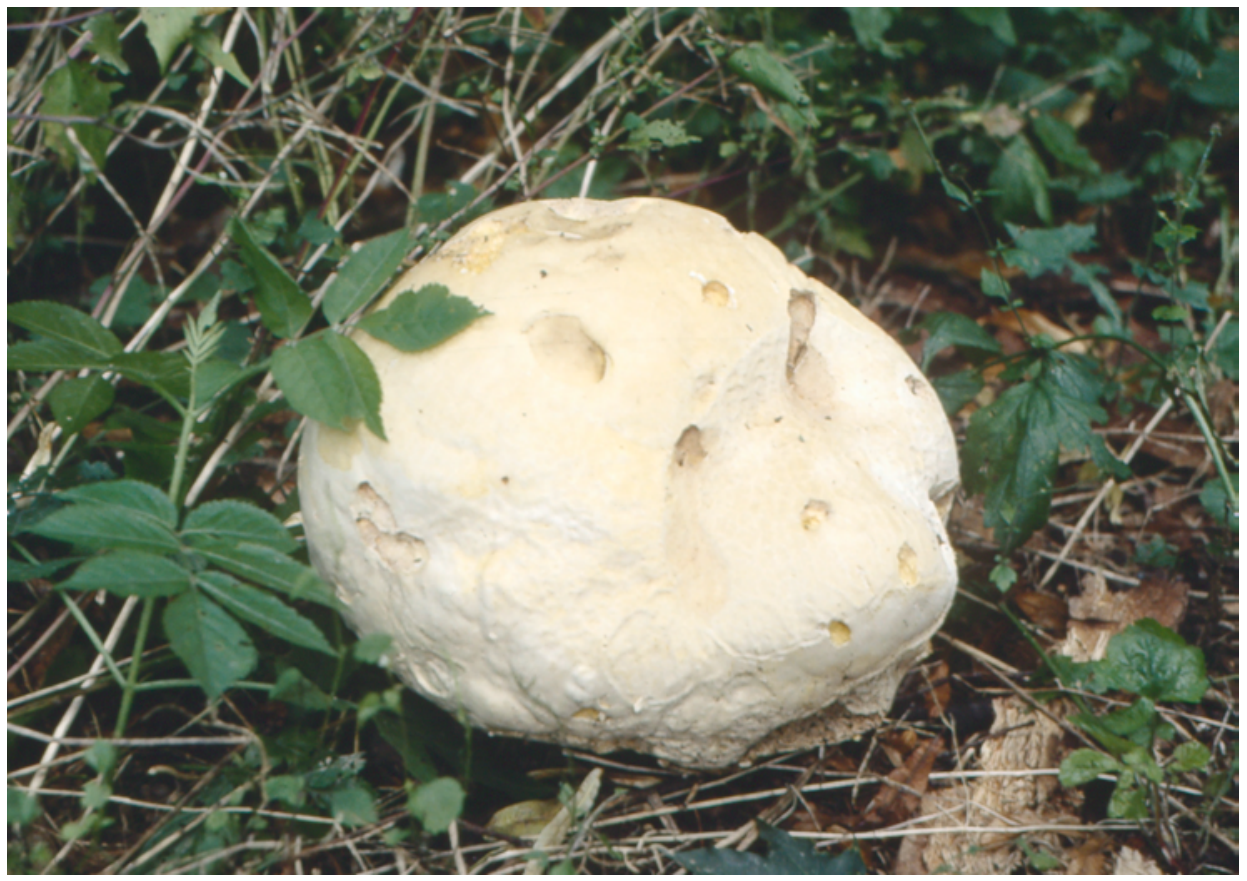

Fig. 5. Langermannia gigantea. Phot. A. Szczepkowski. 


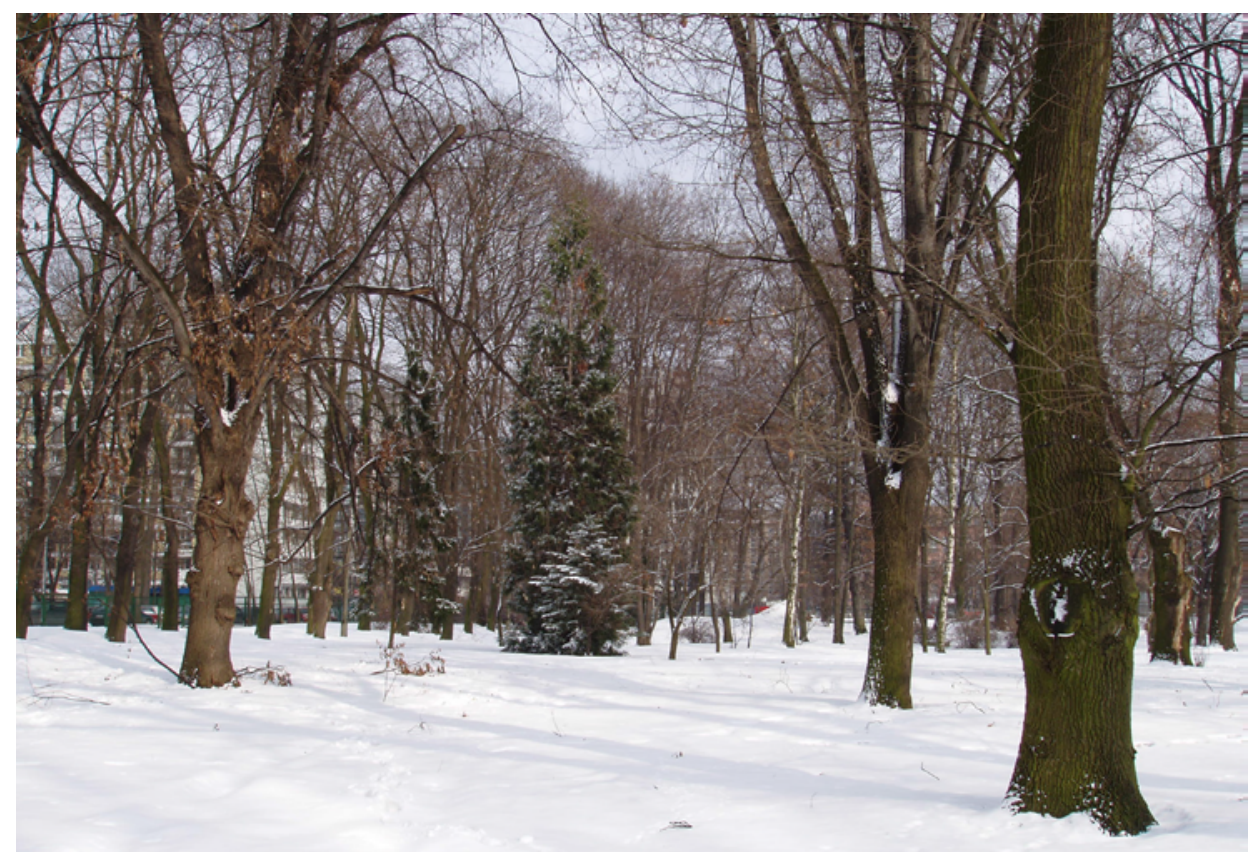

Fig. 2. Snow-covered Dendrological Park. Phot. A. Szczepkowski.

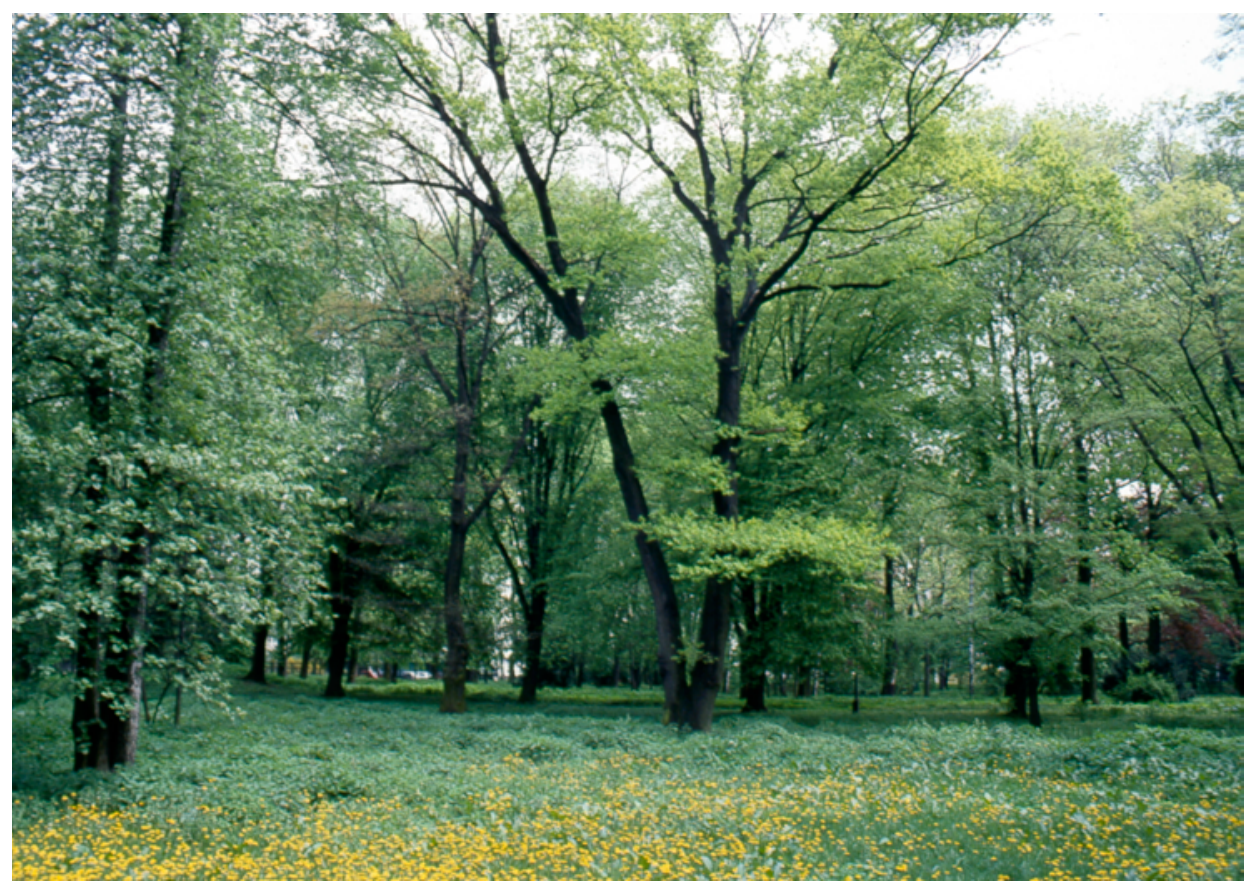

Fig. 3. The largest glade, formed after the removal of dying trees in the Dendrological Park, and its surrounding area. Phot. A. Szczepkowski. 


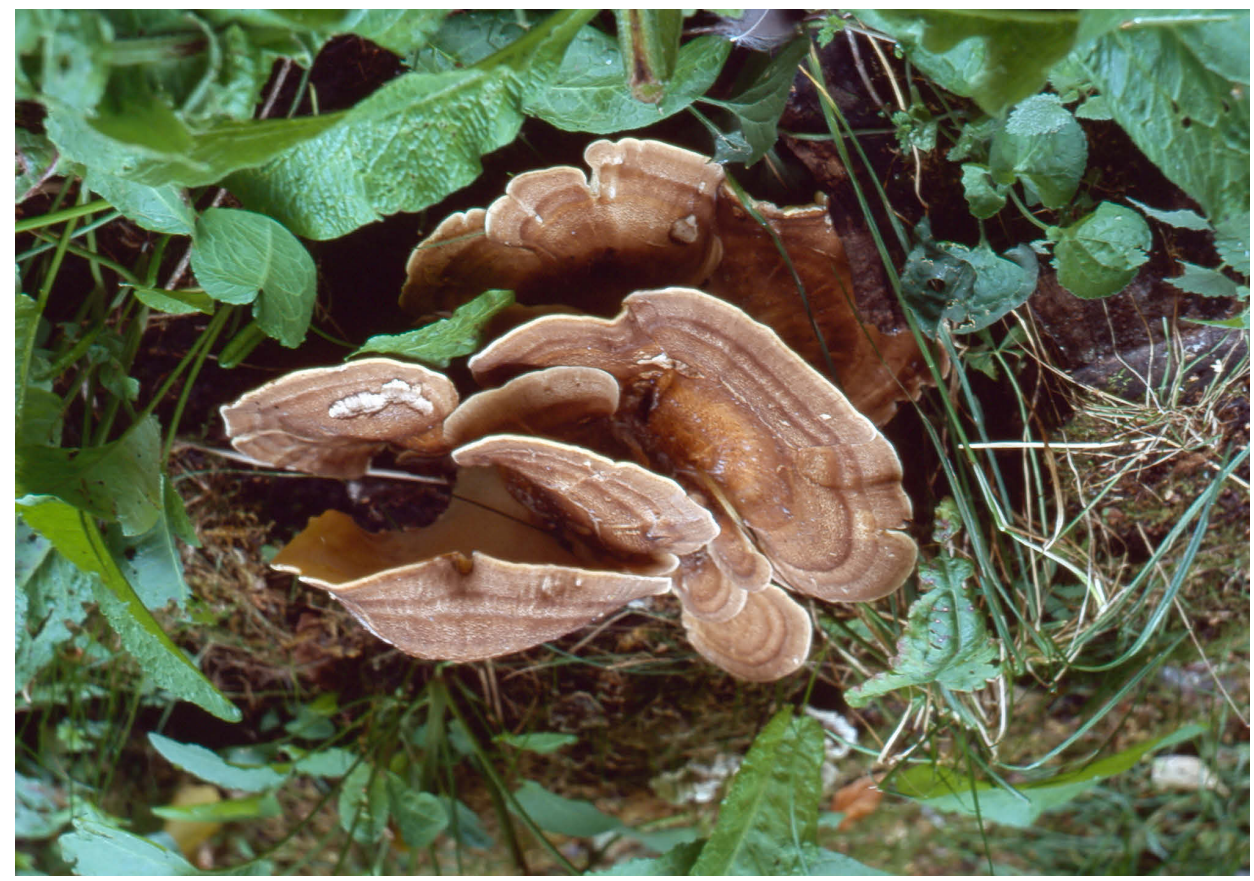

Fig. 6. Meripilus giganteus. Phot. A. Szczepkowski.

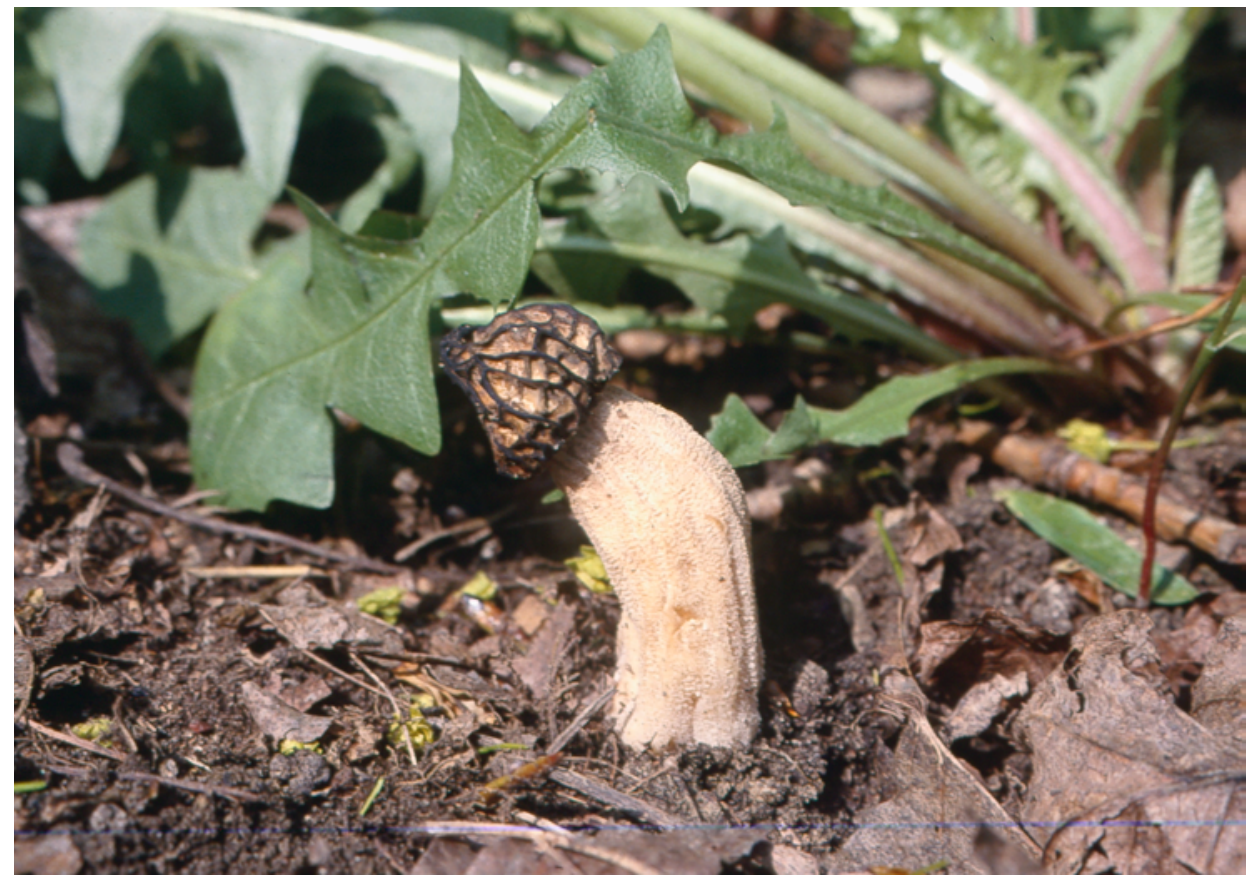

Fig. 7. Morchella semilibera. Phot. A. Szczepkowski. 